\section{ÉTUDE SUR LES BARRHGES EN MHÇONNERIE et Murs de Réservoirs}

pour rendre aptes, aux multiples usages auxquels elles s'appliquent, les eaux qui coulent à la surface du sol, l'homme est obligé de contenir cet élément fluide entre des parois résistantes et étanches. Ces parois peuvent s'appeler cloisons, murs, digues ou barrages, etc.; leur forme, leur nature et leurs dimensions varient avec la diversité des cas qu'on se propose de réaliser : récipients quelconques, réservoirs, canaux d'irrigations ou de navigation, canaux d'amenée d'usines hydrauliques, etc., mais le principe général qui les régit est le même pour tous: ils doivent, par leur construction, être capables de résister à la poussée de l'eau.

L'étude de pareils ouvrages peut être considérée comme un cas particulier de celle des murs de soutènement et elle rentre dans le cadre général de l'étude de la stabilité des constructions. Toutefois, il y a lieu de prendre certaines précautions particulières, d'une part pour assurer l'étanchèité, et d'autre part pour éviter les infiltrations qui, à la longue, pourraient amener la ruine de ces ouvrages.

La rupture de certains barrages ayant arnené des catastrophes retentissantes, on ne saurait apporter trop de soin à l'étude d'un mur de réservoir de grande capacité, cependant, ces catastrophes ne sauraient jeter un discrédit sur les grands barrages, car elles paraissent n'avoir été causées que par la non observation des règles principales formulées en la matière.

Utilité des barrages. - Le but primordial de tout harrage est de créer une retenue d'eau. Cette retenue peut etre variable; alors on cherche surtout à emmagasiner de l'eau au moment où elle est surabondante, pour la restituer à d'autres instants où elle fait défaut. Ou bien, au contraire, cette retenue peut être fixe, et alors on crée une chute d'eau.

Le premier cas est de beaucoup le plus général. Il s'applique aussi bien aux réservoirs des canaux de navigation ou d'irrigations qu'à ceux d'alimentation des villes ou des usines hydrauliques. Il peut lui-même se subdiviser en deux autres cas plus particuliers.

Un réservoir, naturel ou artificiel, peut en effet servir, soit à régulariser le débit d'une rivière pendant le cours d'une année, soit à améliorer le service d'alimentation d'une ville ou le rendement d'une chute d'eau dans l'intervalle d'une journée.

Le débit d'une rivière varie généralement, dans d'assez zrandes proportions, d'une époque de l'année à l'autre, mais il peut être considéré comme pratiquement constant pendant les vingt-quatre heures d'une même journée. Or, à de très rares exceptions près, les besoins d'une ville, ou d'une usine hydraulique, varient avec les moments de la journée; presque nuls pendant certaines heures de la nuit, ils peuvent atteindre des valeurs considérables pendant le jour. Si doncl'on n'a pas de réservoir régulateur, il faudra que le maximum du besoin d'eau soit au plus égal au débit de la rivière qui sera, de la sorte, très mal utilisé. Si, au contraire, l'on dispose d'un réservoir compensateur, on emmagasinera l'eau surabondante pendant la nuit, pour lutiliser pendant le jour, sous forme d'appoint, aux mornents de forte consommation.
Dans le cas d'une usine hydraulique ne desservant qu'une distribution d'éclairage électrique, l'emploi d'un réservoir est tout particulièrement indiqué, car il permet de disposer, pendant quelques heures seulement, d'une puissance bien supérieure à celle de la chute.

Dans ce premier emploi, le réservoir a donc transformé le débit journalier constant d'un cours d'eau en un débit variable à volonté. Mais il peut également jouer le rôle inverse, c'est-à-dire transformer un débit variable en un débit utilisable constant.

Nous avons dit en effet que le débit d'une rivière étail variable suivant les époques de l'année. Or, si les besoins d'une ville ou d'une usine, oscillent entre d'assez larges limites avec les heures d'une même journée, ils varient assez peu, d'une façon générale, dans l'intervalle d'une année. Alors, le besoin maximum d'eau doit être au plus égal au débit minimum de la rivière s'il n'y a pas de résep. voir régulateur.

Ici, le problème est différent suivant qu'il s'agit d'une usine ou d'une ville. Dans le premier cas, l'usine peut, soit créer un réservoir compensateur, soit augmenter la hauteur de sa chute si la nature des lieux le permet, soit installer des machines à vapeur de secours, ou bien créer une succursale en un autre endroit, voir même s'y transporter tout entière. En général, des considérations financières ou économiques dicteront son choix.

Mais il ne peut en être de même pour une ville qứ s'accroît, et lorsque le besoin maximum d'eau aura atteint le minimum de débit du cours d'eau qui l'alimente, il lui faudra impérieusement trouver un moyen d'accroitre ce débit. Et, si elle ne peut disposer de l'eau d'une autri rivière, il lui faudra créer un réservoir afin d'emmagasinêt. l'eau pendant les moments d'abondance pour s'en servir pendant les périodes de disette.

Si la période d'étiage est faible, il suffira d'un réservoir de faible capacité pour obtenir toute l'année un débit rapprochant sensiblement du débit moyen.

Lorsque le niveau de la retenue est fixe, le barrage sert à créer une chute d'eau, ou tout au moins à l'amorcer.

on a quelques fois proposé de construire un barrage de grande hauteur dans le seul but de relever le niveau de l'eau et de créer ainsi la chute. A de rares exceptions prés, cette solution n'est pas à employer, car, en général, il setw plus économique de construire un carsal de dérivation que d'élever un barrage qui coutera fort cher par lui-même êt inondera une surface considérable de terrains qu'il faudrs par suite acquérir.

Mais, le plus souvent, un pareil système de barrage sedt à dériver, dans un canal d'amenée d'usine hydraulique, tout ou partie de l'eau d'une rivière. A cet effet, on construit barrage de faible retenue, submersible pendant les haules eaux, et simplement destiné à dévier la direction de lean et à diriger celle-ci vers les orifices de prise d'eau. All moment des basses eaux, tout le débit de la rivière passtu dans le canal de dérivation.

Les grands barrages peuvent encore jouer un róle bitrfaisant contre les effets dévastateurs des crues en absol: bant celles-ci. En effet, si elles arrivent à la suite d'vin orage, pendant la saison sèche, elles s'emmagasinent d'elles-mêmes. Si l'on a eu soin de maintenir le niveasil maximum normal de la retenue à une certaine hauteur dessous du couronnement, on dispose d'une vaste capacity à la disposition des crues. I,e grand barrage du Furenc 
dont la retenue alimente Saint-Etienne, a été calculé de manière à réaliser ce desideratum. Son auteur, l'ingénieur en chef des Ponts et Chaussées Graëff, avait prévu une tranche d'eau supplémentaire de $5^{\mathrm{m}} 50$ correspondant à l'absorption d'une crue de 400000 mètres cubes, c'est-àdire au double de ce qu'il aurait fallu pour emmagasiner la trombe de 1849 qui inonda Saint-Etienne. Le maximum de la hauteur normale de la retenue avait été prévu de $44^{\mathrm{m}} 50\left(^{\star}\right)$.

Sans s'astreindre à une marge pareille, on peut tout au moins disposer les choses de manière à absorber une notable fraction des crues estimées parmi les plus fortes qu'on ait pu relever depuis un certain nombre d'années, de
Malheureusement, toutes ces matières abandonnées par l'eau s'accumulent sur le fond, qu'elles exhaussent peu à peu, et diminuent de plus en plus la capacité du réservoir. Nous verrons dans un chapitre spécial de quelle manière on peut y remédier.

La photographie ci-jointe représente une vue, prise de l'aval, du barrage d'Avignonnet sur le Drac, pendant sa construction. Ce barrage qui a 20 mètres de hauteur sert à la fois à créer une partie de la chute et à constituer un réservoir régulateur. En effet, la prise d'eau se trouve à 15 mètres au-dessus du fond du Drac. Le barrage crée donc par lui-même une chute de 15 mètres, le surplus sert à créer un réservoir compensateur. La grande vanne que

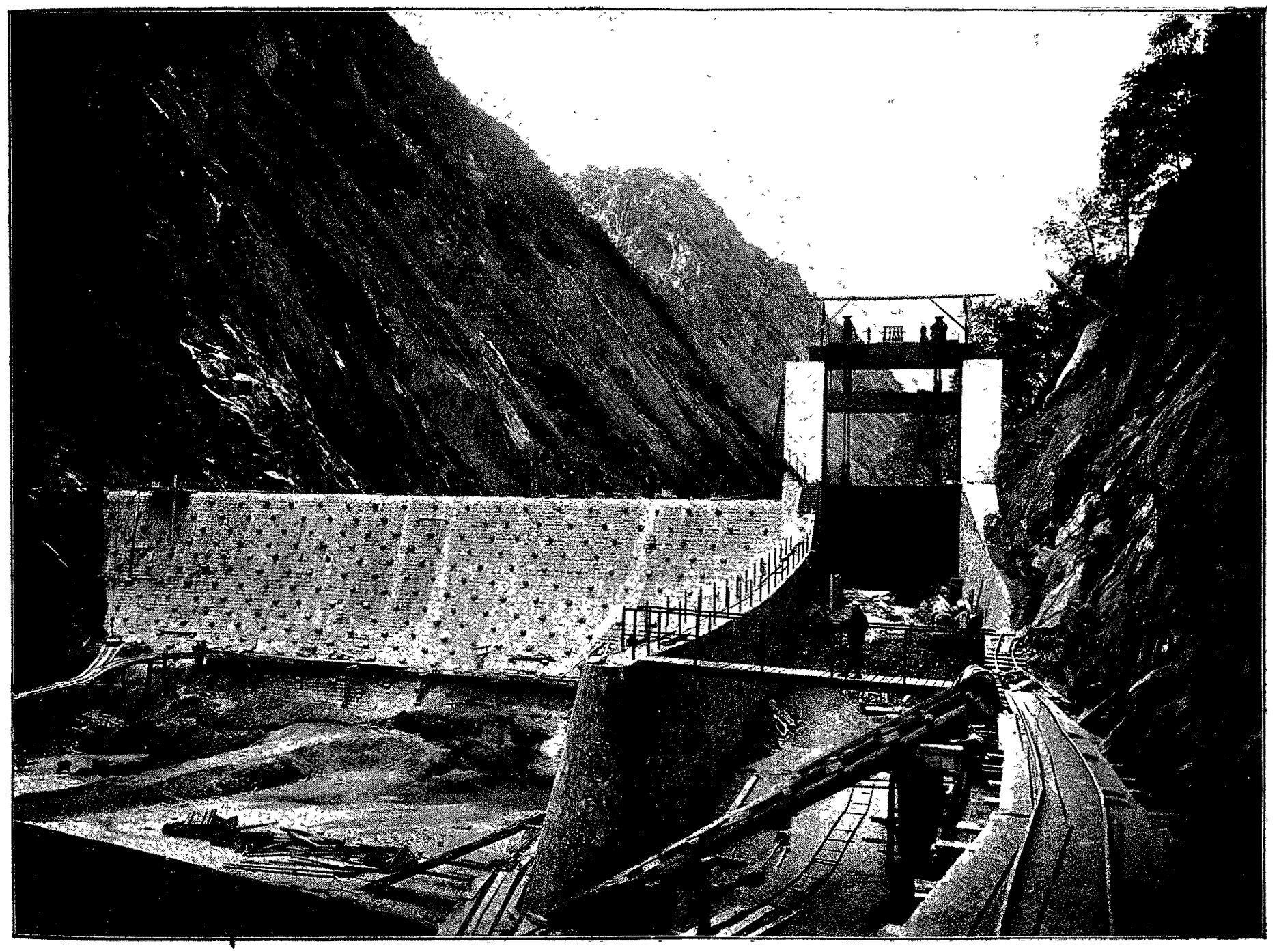

VUE DU BARRAGE D'AVIGNONNET PENDANT SA CONSTRUCTION

manière à atténuer, dans une certaine mesure, leur action dévastatrice.

Dans les installations d'usines hydrauliques on s'attache à obtenir une décantation aussi parfaite que possible, surtout pour les hautes chutes, car les sables, entrainés par l'eau jusque dans les turbines, ont d'abord fait de ronger aubages et distributeurs. On s'impose parfois des dépenses conteuses pour réaliser une décantation toujours imparfaite et, à ce point de vue, les barrages à grande capacité constituent d'immenses chambres de décantation où la vitesse de l'eau est pratiquement nulle. Il en résulte que l'eau $y$ abandonne toutes les matières solides qu'elle tient en suspension et on réalise ainsi une décantation parfaite.

(*) Graer. Barrage du Furens, Annales des Ponts-et-Ghaussćes, 1866. l'on voit à droite de la figure sert à la fois à évacuer les crues et à chasser les graviers. Ce.barrage a été construit par la Société Grenobloise de Force et Lumière pour son usine d'Avignonnet, près de Lamure (Isère). Sa hauteur totale, depuis les fondations jusqu'au couronnement, est de 27 mètres.

\section{CALCUlS ÉlÉMENTAIRES DE STABILITÉ}

Calcul de la poussée, - On désigne généralement sous le nom de poussée l'effort horizontal qui tend à entralner un mur, ou une paroi quelconque, sous l'action de l'eau qui agit sur l'une de ses faces.

On sait que lorsque la hauteur de l'eau est inférieure, ou au plus égale à la hauteur du mur, la valeur de la poussée $F$, exprimée en tonnes par mètre carré de sur- 
face du mur, est représentée par l'expression : $F=0,5 y^{2}$, dans laquelle $y$ représente la hauteur de l'eau exprimée en mètres. Le point d'application de cette poussée est au tiers de la hauteur de l'eau, comptée à partir de la base.

Lorsque la hauteur de l'eau est supérieure à celle du mur, l'expression précédente n'est plus exacte et elle doit etre remplacée par une autre plus générale.

Considérons la section transversale d'un mur ayant à supporter une charge d'eau de $h$ mètres au-dessus de son couronnement (voir figure 1), et découpons, dans la longueur du mur, une tranche verticale de 1 mètre de largeur; soit $\mathrm{AB}$ la projection verticale du parement amont d'une de ces tranches.

La pression de l'eau, exprimée en tonnes par mètre carré, en un point quelconque $\mathrm{M}$ de ce parement $\mathrm{AB}$, est égale à la hauteur de l'eau en ce point et elle est normale à l'élément de surface qui passe par ce point. Si nous désignons par $y$ la distance du point $\mathrm{M}$ au plan horizontal passant par l'arète supérieure $\mathrm{A}$ du mur, et par d $s$ la longueur infiniment petite d'un élément de surface DC, la pression $\subsetneq$, normale à cet élément, aura pour valeur :

$$
q=(y+h) \mathrm{d} s
$$

Cette force $\varphi$ peut se décomposer en deux autres, l'une horizontale $f$, l'autre verticale $p$, et si l'on désigne par $\beta$ l'angle d'inclinaison de l'élément CD sur la verticale, ces forces $f$ et $p$ ont respectivement pour valeur :

$$
\begin{aligned}
& f=\varphi \cos \beta=(y+h) \mathrm{d} y \\
& p=\varphi \sin \beta=(y+h) \mathrm{d} x
\end{aligned}
$$

10 La poussée $F$, c'est-à-dire l'effort horizontal par metre courant dù à l'action de l'eau agissant sur le mur ABB", de hauteur $H$, est la résultante de toutes les forces élémentaires $f$; elle a par suite pour valeur :

$$
F=\int^{I I}(y+h) \mathrm{d} y=\frac{H^{2}}{2}+H h
$$

Pour trouver le point $P$ " d'application de cette force écrivons que le moment de la résultante de l'ensemble des forces $f$ est égal à la résultante des moments de ces forces par rapport à un point quelconque, par rapport au point $B$ par exemple, c'est-à-dire que :

$$
F Z=\int^{H}(y+h)(H-y) \mathrm{d} y=\frac{H^{3}}{6}+\frac{H^{2} h}{2}
$$

$Z$ étant la distance de la force $F$ au joint horizontal BB".

En tirant la valeur de $Z$ de la relation précédente et en la portant dans (1) il vient :

$$
Z=\frac{H}{3} \cdot \frac{H+3 h}{H+2 h}
$$

Lorsque le niveau de l'eau affleure la crète du mur, $h=0$, et l'on retrouve les formules bien connues:

$$
F=\frac{H^{2}}{2} \quad Z=\frac{H}{3}
$$

Si la hauteur $H^{\prime}$ de l'eau devient inférieure à celle du mur, $n$ reste nul dans les équations (1) et (2), mais alors il faut intégrer depuis la hauteur d'eau $y=0$ jusqu'à $y=H^{\prime}$ seulement, de sorte que l'on retrouve encore les formules:

$$
F=\frac{H^{\prime 2}}{2} \quad Z=\frac{H^{\prime}}{3}
$$

Si l'on pose $Z=\mu, H$ on voit que le coefficient $\mu$ est $\mu<\frac{1}{3}$ lorsque la hauteur de l'eau $H^{\prime}$ est inférieure à la hau. teur $H$ du mur, qu'il est $\mu=\frac{1}{3}$ pour $H^{\prime}=H$, et $\mu_{1}>\frac{1}{3}$ pour $H^{\prime}>H$. Lorsque $h$ devient très grand par rapport à $H$, la valeur de $\mu$ tend vers la valeur $\mu=\frac{1}{2}$.

Dans le calcul d'un déversoir on emploiera les formules (1) et (2).

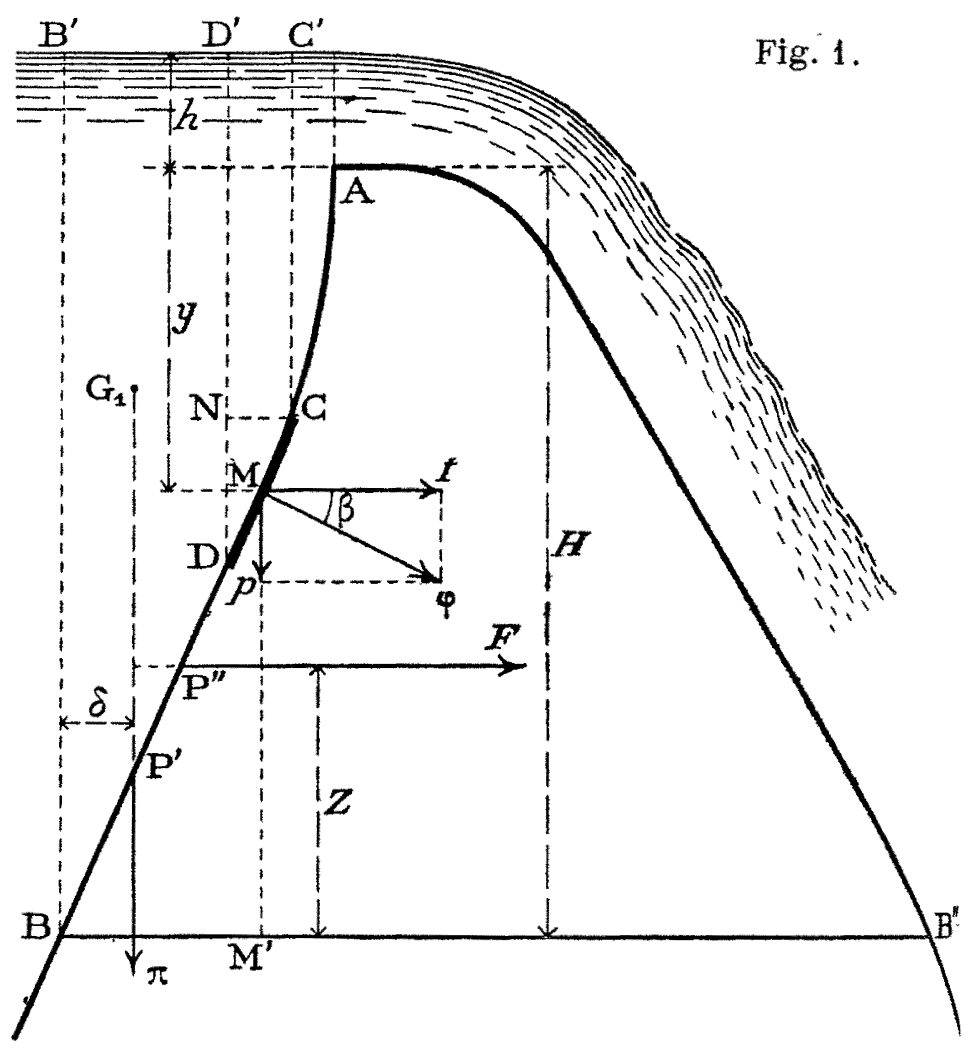

2. La composante verticale $p$ tend, dans une certainé mesure, à contrebalancer l'effet nuisible de l'effort de ren. versement $f$, car elle tend à maintenir le mur sur sa base. Cette force verticale $p$ n'est pas autre chose que le poids de la tranche d'eau infiniment petite D'DCC' qui a précisément pour valeur $(y+h) \mathrm{d} x$, de sorte que l'effort vertical total, que nous désignerons par $\pi$, est égal à la somme des poids des tranches élémentaires D'DCC', c'est-à-dire au poids de l'eau contenue dans le volume B'BAA'. Lepoint P' d'applica: tion de la force $\pi$ se trouve évidemment sur la verticale du centre de gravité $\mathrm{G}_{1}$ du volume B'BAA'.

En résumé, l'action de l'eau sur le parement amont peul être représentée par deux forces : l'une horizontale $F$ ' es'. appliquée en P", l'autre verticale $\pi$ est appliquée en P'.

Loi du trapèze. - Dans l'étude de la stabilité des cons" tructions l'on admet que la pression développée par les forces extérieures est proportionnelle à la déformation produite, et que si la base de l'ouvrage était primitivement plane elle reste plane après la déformation. Cette question étant étudiée en détail dans tous les traités de Résistance des matériaix, nous ne ferons que rappeler les résultats aux* quels on arrive pour le cas particulier qui nous occupe.

Soit $A B C D$ la projection verticale d'un solide prisma. tique, dont le rectangle $\mathrm{C}_{1} \mathrm{D}_{1} \mathrm{D}_{2} \mathrm{C}_{2}$ représente la projection. horizontale, et prenons deux axes rectangulaires passant par les axes principaux de symétrie du rectangle $C_{1} D_{1} D_{2} C_{4}$ Sous l'action d'une force verticale $N$, appliquée en $E_{1}$ la base $\mathrm{CD}$ va prendre la position $\mathrm{CD}^{\prime}$ et si le point $\mathrm{E}$ se trouye 
surl'axe OX, et à une distance $x_{1}$ de l'axe OY, en tout point du rectangle $\mathrm{C}_{1} \mathrm{D}_{1} \mathrm{D}_{2} \mathrm{C}_{2}$ situé sur une parallèle à l'axe OY et d'abscisse $x$, la pression $n$ est représentée par l'équation :

$$
n=\frac{N}{\Omega}\left(1+\frac{12 x x_{1}}{e^{2}}\right)
$$

$e$ étant la longueur de la base $\mathrm{CD}_{1}$ et $\mathrm{C}_{\mathbf{Q}} \mathrm{D}_{2}$ (figure 2). La ligne des pressions nulles est donnée par la relation :

$$
1+\frac{12 x x_{1}}{e^{2}}=0 \quad \text { d'où : } \quad x=-\frac{e^{2}}{12 x_{1}}
$$

d étant la distance du point $\mathrm{E}$ à l'arète $\mathrm{D}_{1} \mathrm{D}_{2}$. Le minimum de pression a lieu avec le minimum de $x$, soit pour $x=-\frac{e}{2}$, c'est-à-dire sur l'arète $\mathrm{C}_{4} \mathrm{C}_{2}$ et sa valeur n' est :

$$
n^{\prime}=\frac{N}{\Omega}\left(1-\frac{6 x_{1}}{e}\right)=\frac{N}{\Omega}\left(\frac{6 d-2 e}{e}\right)
$$

Le maximum de pression a lieu en même temps que le maximum de $x$, soit pour $x=\frac{e}{2}$, c'est-à-dire sur l'arète $\mathrm{D}_{1} \mathrm{D}_{2}$ et cette valeur maximum $n$ " est :

$$
n^{\prime \prime}=\frac{N}{\Omega}\left(1+\frac{6 x_{1}}{e^{2}}\right)=\frac{N}{\Omega}\left(\frac{4 e-6 d}{e}\right)
$$

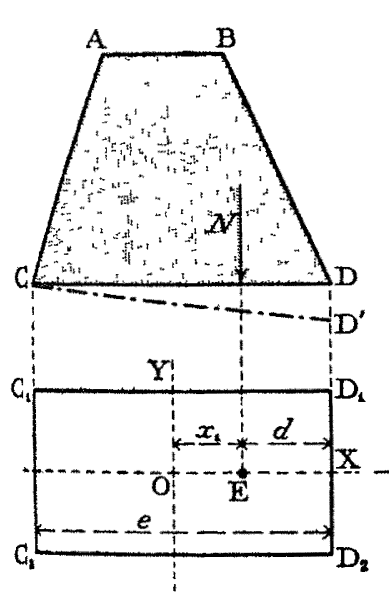

Fig. 2.

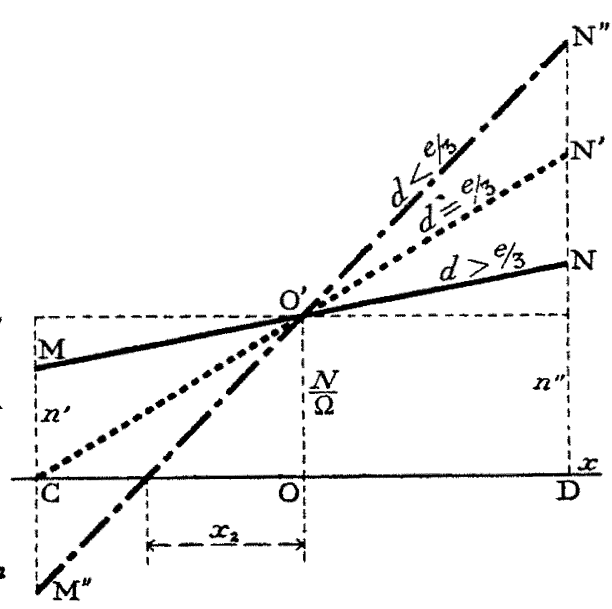

Fig. 3.
La demi-somme $\frac{n^{\prime}+n^{\prime \prime}}{2}$ de la pression minima et de la pression maxima est égale à la pression moyenne $\frac{N}{\Omega}$ Pour toute valeur de $x_{1}<\frac{e}{6}$, c'est-à-dire $d>\frac{e}{3}$, la ligne des pressions nulles se trouve en dehors du rectangle et la pression minima $n$ ' est partout positive.

Pour $x_{1}=\frac{e}{6}$, et $d=\frac{e}{3}$, la ligne des pressions nulles se trouve sur l'arète $\mathrm{C}_{4} \mathrm{D}_{1}$ alors $n^{\prime}=0$ et $n^{\prime \prime}=2 \frac{N}{\Omega}$.

Pour $x>\frac{e}{6}$, et $d<\frac{e}{3}$, la ligne des pressions nulles se trouve à l'intérieur du rectangle, et elle a puur équation :

$$
x=-\frac{e^{2}}{12 x_{1}}
$$

Pour toute valeur de $x$ comprise entre $+\frac{e}{2}$ et $-\frac{e^{2}}{12 x_{1}}$ la pression est positive, et pour toute valeur de $x$ comprise entre $-\frac{e^{2}}{12 x_{1}}$ et $-\frac{e}{2}$ la valeur de la pression est négative, c'est-à-dire qu'il y a traction au lieu d'y avoir compression.
Pour $x_{1}=0, d=\frac{e}{2}$, c'est-à-dire lorsque le point d'application de la force $N$ se trouve au centre de gravité de la base, $n^{\prime}=n^{\prime \prime}=\frac{N}{\Omega}$, et la pression est.partout égale à la pression moyenne.

L'équation (4) est l'équation d'une droite représentant la loi de variation de la pression. L'ordonnée à l'origine 00' de la figure 3 est égale à la pression moyenne et le coefficient angulaire de la droite a pour valeur $\frac{12 N x_{1}}{e^{2} \Omega}$

Pour $x_{1}<\frac{e}{6}$, et $d>\frac{e}{3}$, on obtient la droite MN figurée en trait plein, la pression $n$ est partout positive et plus grande que 0.

Pour $x_{4}=\frac{e}{6}, d=\frac{e}{3}$, on a la droite CN' figurée en traits pointillés, la pression $n$ est nulle en C et égale à $\frac{2 N}{\Omega}$ en N'.

Pour $x_{1}>\frac{e}{6}$ et $d<\frac{e}{3}$, on obtient la droite $\mathrm{M}$ " N" flgurée en traits mixtes; la pression est négative jusqu'à ce que $x=x_{2}=-\frac{e^{2}}{12 x_{1}}$ où la pression est nulle.

Pour $x_{1}=\frac{e}{2}, d=\frac{e}{2}$, la pression est partout égale à la pression moyenne, de sorte que la ligne des pressions est parallèle à l'axe des $x$.

Si l'on transporte les axes de manière qu'ils passent par C, l'équation de la droite devient :

$$
n=n^{\prime}+\left(\frac{n^{\prime \prime}-n^{\prime}}{e}\right) x=P+Q x
$$

La pression variant, dans le cas général, comme les ordonnées des trapèzes CMND ou CM"N"D, on a donné à cette loi de variation le nom de loi du trapèze.

Si l'on découpe dans la longueur du barrage, comme nous l'avons fait précédemment pour le calcul de la poussée, une tranche de 1 mètre de longueur, la section $\Omega$ de la base de cette tranche est égale à $1^{\mathrm{m}} \times e$, de sorte que les valeurs de la pression minima $n$ ' et de la pression maxima $n^{\prime \prime}$ peuvent s'écrire, pour cette tranche :

$$
\begin{aligned}
& n^{\prime}=\frac{N}{e}\left(\frac{6 b-2 e}{e}\right)=\frac{N}{e}\left(6 \frac{b}{e}-2\right) \\
& n^{\prime \prime}=\frac{N}{e}\left(\frac{4 e-6 b}{e}\right)=\frac{N}{e}\left(4-6 \frac{b}{e}\right)
\end{aligned}
$$

Nous avons vu que si $d<\frac{e}{3}$ la pression sur la base devenait négative, c'est-à-dire qu'il y avait traction. Si la maçonnerie travaillant à la traction venait à se rompre, ou bien encore si le massif ABCD reposait simplement sur sa base, sans aucun lien avec son support, la force $N$, au lieu de se répartir sur une base de largeur $e$, se répartirait alors sur une base de largeur telle qu'elle fut partout compriméc, c'est-à-dire sur une largeur égale à $3 d$. La répartition de la pression sur cette nouvelle base se ferait, de nouveau, suivant la loi du trapèze, et, dans ce cas, la pression maximum $n "$ deviendrait $n "=\frac{2 N}{3 d}$.

Conditions de stabilité. - Cherchons maintenant les conditions que doit réaliser un mur pour pouvoir résister à la poussée de l'eau qui agit sur l'un de ses côtés. Nous supposerons dans ce qui va suivre que ce mur est implanté suivant une ligne droite et qu'il ne comporte aucun contrefort ni aucune armature métallique. 
Soit M'MNN' la section transversale d'un pareil mur (fig. 4) et soit $\mathrm{AB}$ la trace, sur le plan de la figure, d'un plan horizontal situé à la profondeur $y$, et coupant cette section transversale.

La partie AMNB du mur doit être en équilibre sous l'action des forces qui agissent sur elle. Ce sont, d'une part la poussée horizontale $F$ de l'eau appliquée au tier's de la hauteur de l'eau à partir de CD, le poids $N$ de la partie AMNB du mur et le poids $\pi$ de l'eau qui appuie sur le parement amont supposé incliné pour plus de généralité, et d'autre part les réactions, tant verticales qu'horizontales, développées sur la section AB par la partie inférieure du mur sur la partie supérieure.

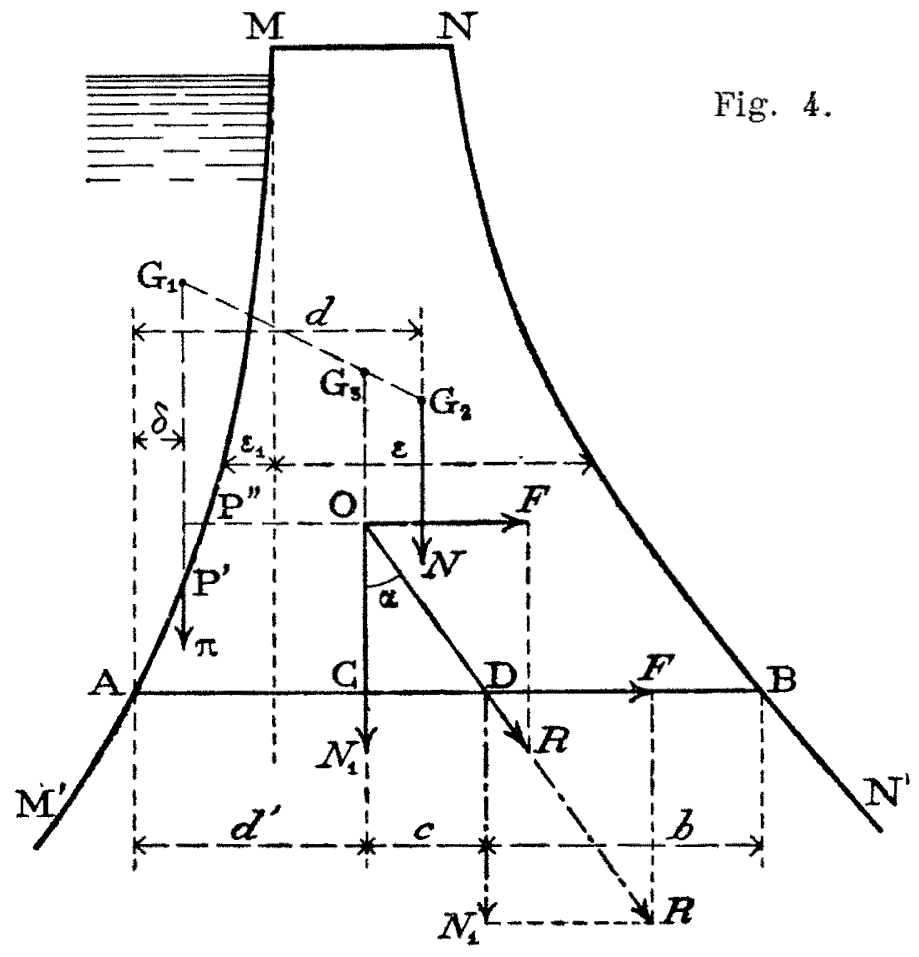

Soit $\mathrm{G}_{1}$ le point d'application de la force $\pi, \delta$ la distance de la direction de cette force au point $A, G_{2}$ le point d'application de la force $N$ et $d$ sa distance au même point A, la résultante $N_{1}$ de $N$ et de $\pi$ est appliquée au centre de gravité fictif $\mathrm{G}_{3}$ et sa distance d' au point A est :

$$
d=\frac{\pi+N d}{\pi+N}
$$

La force verticale $N_{1}$ et la poussée horizontale $F$ se composent entre elles, $a$ leur point de rencontre $O$, pour donner la résultante $R$ qui coupe la base $\mathrm{AB}$ au point $\mathrm{D}$ et qui fait avec $N_{1}$ un angle $\alpha$ tel que $\operatorname{tg} \alpha=\frac{F}{N_{1}}$.

Si l'on appelle $c$ la distance $\mathrm{CD}$ et $b$ la distance du point $D$ au point $B$ il vient :

$$
\begin{gathered}
c=\text { OC } \operatorname{tg} \alpha=\frac{y}{3} \times \frac{F}{N_{1}} \\
b=e-(d+c)
\end{gathered}
$$

Pour que la partie AMNB du mur ne puisse être renversée sous l'action de la poussée $F$, et ne vienne à basculer autour de l'arète $B$, il faut que le moment de renversement de l'ouvrage, c'est-à-dire le moment de $F$ par rapport à B, soit inférieur au moment de stabilité, c'est-à-dire au moment de $N_{1}$ par rapport à $\mathrm{B}$, autrement dit que l'on ait :

ou bien :

$$
N_{1}(b+c)-F \times \frac{y}{3}>0
$$

$$
b>0
$$

Le point de rencontre $\mathrm{D}$ de la résultanle $R$ et de la base $\mathrm{AB}$ ne doit donc jamais sortir de l'intérieur de cette base.

Au point $\mathrm{D}$, de la base $\mathrm{AB}$, la résultante $R$ peut se rede. composer en ses composantes primitives $F$ et $N_{1}$.

La force horizontale $F$ doit être annihilée par la cohésion des maçonneries, et par le frottement exercé sur AB par la pression de la partie supérieure du mur sur la partie infó. rieure. Pour augmenter les conditions de sécurité on ne tient pas compte de la cohésion et l'on suppose que le frot. tement s'oppose seul à la poussée de l'eau. De sorte que, si l'on désigne par $f$ le coefflcient de frottement, l'on doit avoir :

$$
(N+\pi) f \supseteq F \quad \text { ou } \quad \operatorname{tg} \alpha \leq f
$$

a désignant l'angle que fait, avec la normale à $\mathrm{CD}$, la résultante de $N$, de $\pi$ et de $F$.

Si la condition (10) est remplie, la partie AMNB du mur ne pourra pas glisser sur sa base horizontale AB.

La force verticale $N_{1}$ doit être annihilée par la résistance des maçonneries. Or la pression développée par $N_{1}$ en un point quelconque de la base $A B$, peut se déterminer par la loi du trapèze. En appliquant la formule (7) il vient:

$$
n=n^{\prime}+\frac{n^{\prime \prime}-n^{\prime}}{e} x
$$

les $x$ étant comptés à partir du parement amont $A$

M. Maurice Lévy a montré $\left(^{\star}\right)$ que la loi du trapèze cor* respondait bien à la théorie mathématique de l'élasticité dans le cas d'un barrage à profil triangulaire et lorsque la hauteur de l'eıu est au plus égale à la hauteur du barrage, qu'elle n'y correspondait pas rigoureusement dans le cas d'un profil rectangulaire, mais que, dans ce cas, elle accusait des pressions un peu trop fortes à l'aval et un peu trop faibles à l'amont, c'est-à-dire qu'elle donnait des résultals favorables à la sécurité de l'ouvrage.

Lorsque la hauteur de l'eau est supérieure à celle du bar. rage, la loi du trapèze ne correspond plus à la théorie mathématique de l'élasticité et il y a lieu de ne l'appliquer qu'avoc prudence et de se réserver une certaine marge dans la détermination de l'effort limite auquel on fera travaille les matériaux employês.

La pression minima $n$ ' sur le parement amont et la pres. sion maxima $n$ " sur le parement aval sont données parles: relations :

$$
\begin{aligned}
& n^{\prime}=\frac{N_{1}}{e}\left(\frac{6 b-2 e}{e}\right)=\frac{N_{1}}{e}\left(6 \frac{b}{e}-2\right) \\
& n^{\prime \prime}=\frac{N_{1}}{e}\left(\frac{4 e-6 b}{e}\right)=\frac{N_{1}}{e}\left(4-6 \frac{b}{e}\right)
\end{aligned}
$$

MM. Bouvier, Guillemain et Maurice Lévy ont montré que cette valeur de $n$ " ne donnait pas la véritable pression? maxima et que celle-ci devait être :

$$
\begin{array}{ll}
\text { D'après M. Bouvier. } & n{ }_{1}=n "\left(1+\operatorname{tg}^{2} \alpha\right) \\
\text { D’après M. Lévy.... } & n{ }_{1}=n "\left(1+\operatorname{tg}^{2} \beta\right)
\end{array}
$$

$\beta$ étant l'angle que fait le parement aval avec la verticale sur le joint horizontal considéré.

Il faut donc, pour que la maçonnerie du mur ne soit nulle part écrasée, que la pression maxima $n$ ", aussi biell charge qu'à vide, soit inférieure à la résistance limille qu'on assigne pratiquement aux maçonneries. On calculert cette pression maxima de préférence par la méthode Létiji

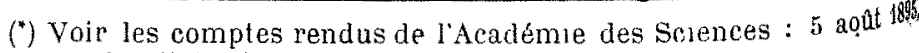
2 mal et 4 juillet 1898. 
qui donne généralement les pressions les plus fortes, et qui de plus est basée sur une théorie plus complète et plus moderne que les autres.

Lorsque l'eau n'agit pas sur le mur, c'est-à dire lorsque celui-ci travaille $a ̀$ vide, les seules forces appliquées au système sont, d'une part le poids $N$ de la maçonnerie AMNB et d'autre part la réaction verticale de la partie du mur située en dessous de $\mathrm{AB}$.

La force verticale $N$ étant appliquée à une distance $d$ du parement amont,la pression minima à l'aval $n$ " et la pression maxima $n$ ' à l'amont ont pour valeur, à vide :

$$
\begin{aligned}
& n^{\prime \prime}=\frac{N}{e}\left(\frac{6 d-2 e}{e}\right)=\frac{N}{e}\left(6 \frac{d}{e}-2\right) \\
& n^{\prime}=\frac{N}{e}\left(\frac{4 e-6 d}{e}\right)=\frac{N}{e}\left(4-6 \frac{d}{e}\right)
\end{aligned}
$$

Il faut éviter que la maçonnerie du parement amont ne travaille a la traction, car, dans ce cas, la moindre fissure introduit l'eau dans l'ouvrage, ce qui produit des sous pressions dont l'effet est de diminuer la stabilité du mur. La stabilité étant diminuée, la fissure ne peut que tendre à s'accroitre, ce qui augmente l'introduction de l'eau et l'effet des sous-pressions et peut amener à la longue la ruine de l'ouvrage. On s'impose généralement la condition que la pression, sur le parement amont, soit au moins égale à zéro, ce qui exige que la résultante passe au moins au tiers de la base à partir du parement aval.

Dans les ouvrages importants on s'attache même à ce que la pression $n^{\prime}$ en charge soit au moins égale à celle de l'eau de manière à empêcher absolument toute introduction d'eau dans la maçonnerie.

Le travail de la maçonnerie à la traction sur le parement aval a moins d'importance que sur l'amont, car ce parement aval n'est pas en contact avec l'eau et le danger des souspressions n'y est pas à craindre,mais comme les maconneries travaillent infiniment moins bien à la traction qu'à la compression il faut éviter de les faire travailler ainsi et lorsque le mur est à vide il est bon, en général, que la force $N$, représentant le poids de la maçonnerie, passe au moins au tiers de la base à partir du parement amont.

Et comme il est préférable de ne pas se tenir sur la limite, on dit que la courbe des pressions doit se maintenir dans le tiers médian de la base.

Sil'on mène la verticale de l'arète $M$ du couronnement du mur et si l'on désigne par $\varepsilon$ la longueur de la partie d'un joint horizontal quelconque qui est comprise entre cette verticale et le parement aval et par $\varepsilon_{1}$ la longueur de la partie du même joint qui est comprise entre celte même verticale et le parement amont, on doit avoir pour chaque mètre courant de longueur du mur :

$$
\begin{gathered}
\pi=\int^{y} y \frac{\mathrm{d} y}{\mathrm{~d} \varepsilon_{1}} \mathrm{~d} y \\
N=K \int^{y}\left(\varepsilon+\varepsilon_{1}\right) \mathrm{d} y
\end{gathered}
$$

$K$ désignant la densité de la maçonnerie exprımée en tounes par mètre cube.

Poids minimum de maçonneries. - Nous avons vu qu'il fallait, pour que la partie $A B C D$ du mur ne puisse glisser sur sa base $\mathrm{AB}$, que l'on ait :

$$
N+\pi \geqslant \frac{F}{f}
$$

Le minimum de $N$ auquel on puisse descendre est évidemment celui qui satisfait à la relation :

$$
N+\pi=\frac{F}{f}
$$

Pour une hauteur d'eau nulle, la poussée $F$ est également nulle, par suite, $(N+\pi)$ peut être théoriquoment égal à zéro.

En remplaçant $N$, $\pi$ et $F$ par leur valeur, et en admettant que le niveau de l'eau atteigne la crète du mur, la relation précédente devient :

$$
K \int^{y}\left(\varepsilon+\varepsilon_{1}\right) \mathrm{d} y+\int^{y} y \frac{\mathrm{d} \varepsilon_{1}}{\mathrm{~d} y} \mathrm{~d} y=\frac{y^{2}}{2 f}
$$

Différentions les deux membres de cette expression par rapport à $y$ et posuns $K f=\operatorname{cotg} \beta$, nous obtiendrons l'équation différentielle :

$$
y \frac{\mathrm{d} \varepsilon_{1}}{\mathrm{~d} y}+K\left(\varepsilon+\varepsilon_{1}\right)=\frac{y}{f}
$$

qui donne la loi suivant laquelle $\varepsilon_{1}$ et $\varepsilon$ doivent croitre en fonction de la hauteur $y$.

Si l'on fait $\varepsilon_{1}=0$, c'est-à-dire si l'on suppose que le parement amont est vertical, il vient, en posant $k f=\operatorname{cotg} \beta$ :

$$
\varepsilon=y \operatorname{tg} \beta
$$

L'épaisseur du mur crott proportionnellement à la hauteur et son profil est un profil triangulaire, l'angle au sommet étant tel que $\operatorname{cotg} \beta=K f$.

Si l'on suppose que les deux parements soient inclinés et que, de plus, pour chaque joint, $\varepsilon_{1}$ soit dans un rapport cons. tant avec $\varepsilon$, de telle façon que $\varepsilon_{1}=\beta \varepsilon$, on a :

$$
\frac{\mathrm{d} \varepsilon}{\mathrm{d} y}+K\left(1+\frac{1}{\mu}\right) \frac{\varepsilon}{y}=\frac{1}{\mu f}
$$

équation différentielle de la forme:

$$
\frac{\mathrm{d} \varepsilon}{\mathrm{d} y}+P \varepsilon=Q
$$

dont l'intégrale générale est:

$$
\varepsilon=e^{-\int P \mathrm{~d} y}\left[\int Q e^{\int P \mathrm{~d} y} \mathrm{~d} y+C\right]
$$

$e$ étant ici la base des logarithmes népériens.

Or, l'on a:

$$
\begin{array}{cc}
\int P \mathrm{~d} y=K\left(1+\frac{1}{\mu}\right) y & \text { et } \int \frac{\mathrm{d} y}{y}=K\left(1+\frac{1}{\mu}\right) \mathrm{L}(y) \\
e^{-\int P \mathrm{~d} y}=y^{-K\left(1+\frac{1}{\mu}\right)} & \text { et } e^{\int P \mathrm{~d} y}=y^{K\left(1+\frac{1}{\mu}\right)}
\end{array}
$$

L'intégrale générale est donc:

$$
\varepsilon=y^{-K\left(1+\frac{1}{\mu}\right)}\left\{\frac{1}{\mu f\left[K\left(1+\frac{1}{\mu}\right)+1\right]} y^{K\left(1+\frac{1}{\mu}\right)+1} C+\right\}
$$

Comme théoriquement pour une hauteur d'eau nulle $(y=0)$, on peut avoir une épaisseur nulle au sommet $(\varepsilon=0)$, la constante $C$ est nulle et il reste:

$$
\begin{gathered}
\varepsilon=\frac{y}{\mu \cdot f\left(1+K\left(1+\frac{1}{\mu}\right)\right)}=y \operatorname{tg} \beta^{\prime} \\
\varepsilon_{1}=\mu \varepsilon=y \operatorname{tg} \beta^{\prime} \\
\varepsilon+\varepsilon_{1}=y\left(\operatorname{tg} \beta^{\prime}+\operatorname{tg} \beta^{\prime \prime}\right)
\end{gathered}
$$

L'épaisseur du mur croít encore proportionnellement à la hauteur et le profil est encore un profil triangulaire. 
En remplaçant $\operatorname{tg} \beta^{\prime}$ et $\operatorname{tg} \beta^{\prime \prime}$ par leurs valeurs on trouve :

$$
\varepsilon+\varepsilon_{1}=\frac{y}{f\left(K+\frac{\mu}{1+\mu}\right)}>\frac{y}{K f}
$$

Le minimum de $\left(\varepsilon+\varepsilon_{1}\right)$ a lieu lorsque $\mu_{1}=\infty, \varepsilon=0$, c'està-dire lorsque le parement aval est vertical.

L'épaisseur du mur est alors:

$$
\varepsilon_{1}=\frac{y}{f(K+1)}
$$

Ainsi donc, si l'on se plaçait simplement au point de vue du glissement suivant une direction horizontale, le profil conduisant au minimum de maçonneries serait un profil triangulaire à parement amont incliné et à parement aval vertical. De prime abord, ceci parait assez rationnel, car le poids de l'eau qui agit sur le parement amont incliné tend à s'opposer au renversement du mur et à maintenir louvrage sur sa base,et il semble que plus le poids de cette eau sera grand mieux la stabilité sera assurée; aussi, se basant sur cette remarque, quelques auteurs ont préconisé l'emploi d'un parement amont incliné. Mais, lor'sque l'on applique les formules (8), (9), (11) et (12) pour calculer le travail des maçonneries, l'on s'aperçoit que les meilleures conditions de stabilité sont au contraire réalisées lorsque le parement amont est vertical. Tous les ingénieurs qui ont eu à calculer des barrages savent que la condition (10) de non glissement suivant une direction horizontale est toujours satisfaite lorsque les conditions de travail des maçonneries sont réalisées. Il s'ensuit que le véritable profil minimum sera le profil triangulaire à parement amont vertical qui remplit ces dernières conditions, ainsi que nous allons le montrer dans le paragraphe suivant.

Profil triangulaire. - Ainsi que nous vellons de le voir, c'est le profil triangulaire qui réalise le plus économiquement la condition de non renversement des murs ayant à supporter une charge d'eau.

Soit ABC la section transversale d'un mur de barrage à profil triangulaire dont le parement amont est incliné et $\mathrm{P}$ le pied de la verticale abaissée du sommet A sur la base BC. Désignons par $m$ le segment $\mathrm{BP}$, par $m$ 'le segment $\mathrm{PG}$, par $e$ la largeur de la base et par $K$ la densité des maçon-

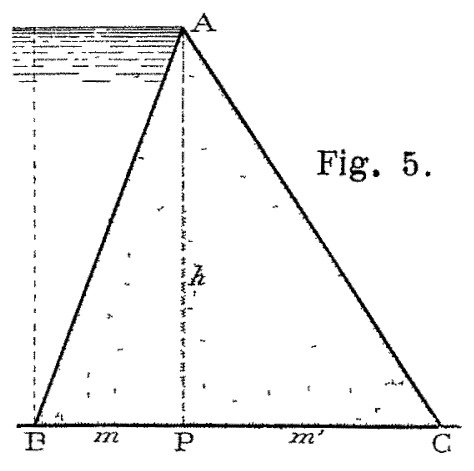
neries. Pour simplifier les calculs nous admettrons que le niveau de l'eau affleure le sommet $\mathrm{A}$. Dans ces conditions l'on a :

$$
\begin{aligned}
& \pi=\frac{m y}{2} \quad N=K\left(m+m^{\prime}\right) \frac{y}{2}=K \frac{y e}{2} \\
& N_{1}=\frac{y}{2}(m+K e) \\
& \operatorname{tg} \alpha=\frac{F}{N_{1}}=\frac{y}{m+K e} \\
& N_{1} d=\frac{m y}{2} \times \frac{m}{3}+\frac{K m y}{2} \times \frac{2 m}{3}+\frac{K m^{\prime} y}{2}\left(m+\frac{m^{\prime}}{3}\right) \\
& d^{\prime}=\frac{1}{3} \frac{m^{2}+K e(m+e)}{m+K e} \\
& c=\frac{y}{3} \times \frac{F}{N_{1}}=\frac{y^{2}}{3(m+K e)}
\end{aligned}
$$

$$
b=e-\frac{m^{2}+K e(m+e)+y^{2}}{3(m+K e)}
$$

en appliquant la loi du trapèze il vient :

$$
\begin{gathered}
n^{\prime}=\frac{y}{2}\left(\frac{m+K e}{e}\right)\left[\frac{6 e}{e}-\frac{6\left(m^{2}+y^{2}+K e(m+e)\right.}{3 e(m+K e)}-2\right] \\
n^{\prime}=\left(K-\frac{y^{2}}{e^{2}}\right) y-\frac{y m}{e^{2}}[m+e(K-2)] \\
n^{\prime \prime}=\frac{y}{2}\left(\frac{m+K e}{e}\right)\left[4-\frac{6 e}{e}+\frac{6\left(m^{2}+y^{2}+K e(m+e)\right.}{3 e(m+K e)}\right] \\
n^{\prime \prime}=\frac{y^{3}}{e^{8}}+\frac{y m}{e^{8}}[m+e(K-1)]
\end{gathered}
$$

Si le parement amont est vertical :

$$
\begin{gathered}
n^{\prime}=\left(K-\frac{1}{\operatorname{tg}^{2} \beta}\right) y \\
n^{\prime \prime}=\frac{y}{\operatorname{tg}^{2} \beta}
\end{gathered}
$$

$\beta$ étant l'angle d'inclinaison du parement aval sur la verticale.

On voit que dans ce cas la compression de la maçonnerie sur le parement aval croît proportionnellement à la hauteur; il ell est de même pour la pression à l'amont.

Il est à remarquer que l'expression de $n$ " est indépen. dante de $K$, il en résulte que deux murs ayant même pro. fil, mais constitués de matériaux de densités différentes, auront même valeur de $n^{\prime \prime}$.

Pour que la pression $n^{\prime}$ à l'amont ne soit jamais néga. tive il faudra que :

$$
K-\frac{1}{\operatorname{tg}^{2} \beta}>0
$$

le minimum d'inclinaison du parement aval est donc donné par la relation :

$$
\operatorname{tg} \beta=\sqrt{\frac{1}{K}}
$$

$K$ étant compris entre 2 et 2,5 pour les barrages en maçonnerie, la valeur minimum de $\operatorname{tg} \beta$ est comprise entre 0,0 : (pour $K=2$ ) et 0,632 (pour $K=2,5$ ).

Pour que cette même pression $n$ ' soit égale à la pression de l'eau il faut que :

d'où :

$$
\begin{aligned}
& K-\frac{1}{\operatorname{tg}^{2} \beta}=1 \\
& \operatorname{tg} \beta=\sqrt{\frac{1}{K-1}}
\end{aligned}
$$

ce qui correspond à une inclinaison de $1^{\mathrm{m}}$ par mètre pour $K=2$ et de 0 m 816 pour $K=2,5$.

La valeur-de $b$ peut s'écrire :

$$
b=\frac{y}{3}\left[2 \operatorname{tg} \beta-\frac{1}{K \operatorname{tg} \beta}\right]
$$

Cette équation est celle d'une droite. La cour.ue des pres sions, lieu géométrique des points situés à une distance du parement aval, est donc une droite issue du sommet du triangle.

Les méthodes de MM. Bouvier et Maurice Lévy (formules 11 et 1\%) donnent, pour la valeur de la compression maxima maximorum :

$$
\left.\begin{array}{c}
n_{1}=\frac{y}{t g^{2} \beta}\left(1+\frac{1}{K^{2} t^{2} \beta}\right) \\
n_{2}{ }^{\prime \prime}=y\left(1+\frac{1}{\operatorname{tg}^{2} \beta}\right)
\end{array}\right\}
$$


Cette valeur est plus forte avec la méthode de M. Maurice Lévy qu'avec celle de M. Bouvier.

Si p est la limite pratique de résistance des maçonneries àla compression il faudra, pour une hauteur donnée $y$, que linclinaison du parement aval soit telle que :

$$
\operatorname{tg}^{2} \beta \supseteq \frac{y}{\rho-y}
$$

pour $y=50 \mathrm{~ms}$ et $\rho=100$ tonnes par mètre carré, soit 10 kilogs par centimètre carré, $\operatorname{tg} \beta=1$ et le parement aval doit être incliné à $45^{\circ}$.

Si le parement amont n'est pas vertical, $n$ et $n$ " sont données par les formules (15) et (16) et l'on voit immédiatement que $n$ " est toujours plus grand dans ce cas que dans celui du parement vertical et qu'il l'est d'autant plus que $m$ est plus grand.

On voit également que $n$ 'est, pour les murs en maçonnerie, toujours plus petit que dans le cas du parement vertical.

Il y a donc avantage à maintenir le parement amont vertical tant que la pression à l'amont, à vide, n'atteint pas la limite pratique de résistance des maçonneries à la compression.

La valeur de $b$ peut s'écrire:

$$
b=y\left[\mu-\frac{\lambda^{2}+K \mu(\lambda+\mu)+1}{3(\lambda+K \mu)}\right]
$$

en posant : $\quad e=\mu y \quad$ et $m=\lambda y$.

La courbe des pressions en charge est encore une droite passant par le sommet du triangle.

A vide, lorsque le parement amont est vertical, la courbe des pressions est verticale et elle passe toujours par le tiers de la base de telle sorte que :

$$
n^{\prime \prime}=0 \quad \text { et } \quad n^{\prime}=2 \frac{N}{e}=K y
$$

Lorsque le parement amont est incliné, la courbe des pressions, qui est encore une droite, passe toujours dans le tiers médian et l'on a :

$$
\begin{gathered}
d=\frac{e+m}{3}=y\left(\frac{\mu+\lambda}{3}\right) \\
n^{\prime}=K y\left(1-\frac{m}{e}\right) \\
n^{\prime \prime}=K y \cdot \frac{m}{e}
\end{gathered}
$$

Profil rectangulaire. - Nous avons vu que l'épaisseur all sommet d'un barrage pouvait être théoriquement nulle, mais il ne saurait en être ainsi pratiquement. Tout d'abord, la partie supérieure d'un pareil unur serait impossible à construire; d'autre part, l'épaisseur au sommet doit être assez. forte pour résister au choc des vagues ou des corps flottants, voir même à la pression de la glace dans le cas d'un canal de peu de largeur; parfois aussi le couronnement doit en même temps servir au passage des piétons ou des voitures, il en résulte que le haut d'un mur doit avoir la forme d'un trapèze ou d'un rectangle.

Nous allons étudier tout d'abord le cas d'un profil rectangulaire. En appliquant la loi du trapèze, on trouve :

$$
\begin{aligned}
\pi=0 \quad N_{1} & =N=K e y \\
\operatorname{tg} \alpha=\frac{F}{N_{1}} & =\frac{y}{2 K e}
\end{aligned}
$$

$$
\begin{gathered}
d^{\prime}=\frac{e}{2} \quad c=\frac{y^{2}}{6 K e} \quad b=\frac{e}{2}-\frac{y^{2}}{6 K e} \\
n^{\prime}=\left(K-\frac{y^{2}}{e^{2}}\right) y \\
n^{\prime \prime}=\left(K+\frac{y^{2}}{e^{2}}\right) y
\end{gathered}
$$

Si l'on compare les formules (24) et (25) avec les formules (17) et (18) du profil triangulaire avec parement vertical à l'amont, on voit que, pour une même hauteur $y$ et une même base $e$, la pression à l'amont $n$ ' a la même valeur dans les deux cas. Mais, pour arriver à ce résultat, le profil rectangulaire emploie un volume de maçonnerie double de celui du triangle.

On voit également que la pression $n$ " à l'aval est supérieure à celle du triangle de la quantité $K y$.

Pour que la pression à l'amont $n$ ' ne soit pas négative, il faut que $e$ soit au moins égal à $\frac{y}{\sqrt{K}}$; pour que cette pression soit égale à celle de l'eau il faut que :

$$
K-\frac{y^{2}}{e^{2}}=1 \quad e=\frac{y}{\sqrt{K-1}}
$$

En se reportant à l'équation qui donne la valeur de $b$, on voit que la courbe des pressions est une parabole dont le paramètre a pour valeur $3 \mathrm{Ke}$ et qui passe par le milieu du couronnement de l'ouvrage.

Les méthodes de MM. Bouvier et Maurice Lévy donnent, dans ce cas, pour la valeur de la pression maxima maximorum :

$$
\left.\begin{array}{c}
n_{1}=\left(K+\frac{y^{2}}{e^{2}}\right)\left(1+\frac{y^{2}}{4 K^{2} e^{2}}\right) y \\
n_{2}=n^{\prime \prime}=\left(K+\frac{y^{2}}{e^{2}}\right) y
\end{array}\right)
$$

Eu comparant les formules (21) et (24) on voit que la pression maximorum, calculée par la méthode de M. Maurice Lévy est, dans le cas du rectangle, plus forte de la quantité $(K-1)$ y que dans le cas du triangle à parement amont vertical.

Avec la méthode Bouvier cette différence est :

$$
y\left[K-\frac{1}{4 K} \cdot \frac{y^{2}}{e^{2}}\left(\frac{3}{K} \cdot \frac{y^{2}}{e^{2}}-1\right)\right]
$$

Sa limite inférieure doit avoir lieu pour $\frac{y^{2}}{e^{2}}=K$, puisque pour celte valeur $n^{\prime}=0$. Elle est alors égale à $\left(K-\frac{1}{2}\right) y$.

on voit que la pression maxima maximorum est, avec ces méthodes, plus forte pour le rectangle que pour le triangle.

A vide, la courbe des pressions n'est pas autre chose que la verticale médiane $d=\frac{e}{2}$, par suite :

$$
n^{\prime}=n^{\prime \prime}=\frac{N^{-}}{e}=K y
$$

La pression à vide est partout égale à la plus forte pression du profil triangulaire.

Ainsi donc, le profll rectangulaire est beaucoup plus coúteux que le profil triangulaire et il donne des résultats moins favorables. On ne devra donc l'employer que lorsque des circonstances particulières l'exigeront.

Profil trapézoïdal. - Ce profil est intermédiaire entre le profil rectangulaire et le profil triangulaire. Son parement 
amont peut être vertical ou incliné. Nous allons étudier ce profil dans le cas où le parement amont vertical (fig. 6) en supposant, pour simplifier, que le niveau de l'eau affleure la crête du mur.

Désignons par $e_{0}$ la largeur du couronnement, on a respectivement :

$$
\begin{gathered}
\pi=0 \quad N_{1}=N=K \dot{y} \frac{\left(e_{0}+e\right)}{2} \\
d^{\prime}=\frac{e_{0} y \times \frac{e_{0}}{2}+\frac{\left(e-e_{0}\right) y}{2} \times\left(e_{0}+\frac{e-e_{0}}{3}\right)}{\left(\frac{e_{0}+e}{2}\right) y} \\
\operatorname{tg} \alpha=\frac{F}{N_{1}}=\frac{y\left(e_{0}^{2}+e_{0} e+e^{2}\right.}{3\left(e_{0}+e\right)} \\
b=e-\frac{K\left(e_{0}^{2}+e\right)}{3 K\left(e_{0}+e\right)} c=\frac{y^{2}}{3 K\left(e_{0}+e\right)} \\
n^{\prime}=\left[K\left(1+\frac{e_{0}}{e}-\frac{e_{0}^{2}}{e^{2}}\right)-\frac{y^{2}}{e^{2}}\right] y \\
n^{\prime \prime}=\left[K \frac{e_{0}^{2}}{e^{2}}+\frac{y^{2}}{e^{2}}\right] y
\end{gathered}
$$

Si l'on fait $e_{0}=0$ (cas du triangle) on retrouve les formules (17) et (18). Si l'on fait $e_{0}=e$ (cas du rectangle) on retrouve les formules (24) et (25).

En comparant entre elles ces diverses formules on voit que, pour une même base $e$ et une même hauteur $y$, la pression $n$ 'à l'amont est plus forte dans le cas du trapèze

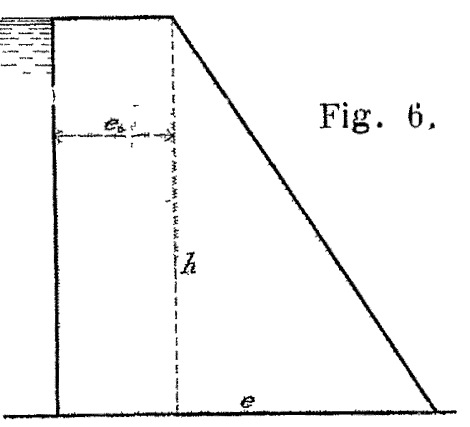
que dans les cas du triangle et du rectangle. La différence qui est égale à $\frac{e_{0}}{e}\left(1-\frac{e_{0}}{e}\right)$ est maximum lorsque $e_{0}=\frac{e}{2}$.

La pression $n " \dot{a}$ l'aval est plus forte dans le cas du trapèze que dans le cas du triangle mais "elle est moins forte que dans le cas du rectangle.

La pression maximum maximorum calculée par la méthode de $\mathrm{M}$. Maurice Lévy a pour valeur :

$$
n "={ }_{2}\left(K \frac{e_{0}^{2}}{e^{2}}+\frac{y^{2}}{e^{2}}\right)\left[1+\left(\frac{e-e_{0}}{y}\right)^{2}\right] y
$$

Sa limite inférieure est $\frac{y^{3}}{e^{2}}+y \quad$ (cas du triangle);

$$
\text { Sa limite supérieure est } \frac{y^{3}}{e^{2}}+K y \text { (cas du rectangle). }
$$

Elle est donc plus forte que pour le triangle et moins forte que pour le rectangle. Il est facile de voir qu'il en est de même avec la méthode Bouvier.

Le profil trapézoïdal, considéré comme mur de retenue est donc préférable au profil rectangulaire, puisqu'il donne des valeurs plus grandes pour la pression à l'amont $n$ ' et moins grandes pour les pressions $n$ " et $n^{\prime \prime}{ }_{1}$ à l'aval.

$$
\begin{array}{cc}
\text { A vide on } \mathrm{a}: & d=d^{\prime} \\
n^{\prime}=K\left[1+\frac{e_{0}}{e}-\frac{e_{0}{ }^{2}}{e^{2}}\right] y \\
n^{\prime \prime}=K \frac{e_{0}^{2}}{e^{2}} y
\end{array}
$$

La pression maxima à vide est un peu plus forte que pour le triangle et pour le rectangle. La différence qui est égale à $\frac{e_{0}}{e}\left(1-\frac{e_{0}}{e}\right)$ est maxima lorsque $\frac{e_{0}}{e}=\frac{1}{2}$; dans ce cas parti. culier elle est 1,25 Ky.

(A suivre.)

H. BELLET.

\section{LE MOIS HYDRO-ÉLECTRIQUE}

\section{ACADÉMIE DES SCIENCES}

\section{MÉGANIQUE ET ÉLECTRIGITÉ}

Faculté que le béton armé possède de supporter de grand's allongements. - Note de M. Considère, séance du 30 janvier,

Dans trols communications faites à l'Académie, le 2 décembre 1898 , le 2 janvier 1899 et le 18 août 1902 , j'ai rendu compte d'expériences qui démontrent les faits suivants :

"Lorsqu'on soumet des pièces en béton armé à la traction simple ou à la flexion, les fibres tendues se comportent comme si elles n'étaient pas armées tant que leur tension et leur allongement ne dépassent pas ceux que le béton non arme peut supporter sans se rompre. Si l'épreuve est poussée plus loin, le béton, armé et préparé comme il convient, peut supporter des allongements beaucoup plus forts pendant lesquels sa tension reste sensiblement constante et, par suite, son module d'élasticité est nul.

Ces faits, acceptés par nombre d'ingénieurs comme base de la théorie et du calcul des constructions armées, ont été accueillis par d'autres avec incrédulité, malgré l'explication que j'en avass donnée dans la communication du 2 janvier 1893 . Des savants allemands et américains ont fait des expériences de contrôle; ils ont observé que les pièces armées, fabriquées sous leur direction, se fissuraient dès qu'elles subissaient des allongements sous lesquels se brise le béton non armé; ils ont décrit les moyens employés par eux pour déceler les fissures capillaires et la conclusion qui ressort, implicitement au moins, de leurs publications est que, dans mes expériences aussi, il a dû se produire des fissures que je n'ai pas réussi à apercevoir.

Pour trancher cette question, qui a une sérieuse importance au point de vue de la théorie du béton armé et aussi des propriétés générales des corps qui ont une constitution moléculaire analogue à celle des bétons et mortiers, j'ai cru utile de faire de nouvelles expériences. Elles ont été exécutées au labo. ratoire de l'Ecole des Ponts et Chaussées, sous la direction de $M$. Mesnager, avec la collaboration de M. Mercier.

Des ouvriers de M. Grouselle, entrepreneur de béton armé, ont fabriqué deux poutres de 3 mètres de longueur et de la section ci-contre en employant les procédés pratiques dont ils avaient l'habitude et sans prendre de précautions spéciales.

Le béton renfermait 400 kilogr. de ciment de Portland de la marque E. Candlot et Cie pour 400 litres de sable et 800 litres de gravier calcaire. La quantité d'eau employée pour le gâchage representait $9,6 \%$ du poids des matières sèches.

L'armement de la poutre consistait en deux barres d'acier doux de 16 millimètres de diamètre et trois barres de 12 millimètres placées près de la face qui devait subir les plus grands allongements.

L'une de ces poutres a été conservée à l'air et couverte de sacs vides et de planches qu'on arrosait fréquemment pendant le premier mois et tous les deux jours ensuite; l'autre a été immergée après un jour de durcissement et conservée sous l'eau.

Ces poutres, fabriquées les 27 et 28 mal 1904, ont été essayées par Hexion les 21 novembre et 22 décembre. Posées sur des appuis distants de om o5 de leurs extrémités, elles ont subi la pression d'un appareil hydraulique en deux points placés symétriquement à $0^{\mathrm{m}} 70$ de part et d'autre du milieu, de sorte que, sur une longueur de $1^{\mathrm{m}} 40$, le moment de flexion était constant et l'effort tranchant nul.

Au milieu de cette longueur, on a observé les allongements de la face tendue sur une longueur de $\mathrm{Im}_{\mathrm{m}}$, au moyen de deux micros" copes montés sur une même tige et l'on a mesuré les raccourcissements de la partie comprimée sur une longueur de o ${ }^{\mathrm{m}} 5 \mathrm{o}$ au moyen de deux appareils Manet-Rabut placés sur les faces latérales.

Conformément au plan fixé d'avance, on a arrêté le chargement au moment où l'allongement des fibres extrêmes du béton étail de $o^{m m} 625$ pour la première poutre et de $1^{\mathrm{mm}} 300$ pour la seconde. $0 \mathrm{n}$ n'apercevait alors, au microscope, aucune fissure dans l'enduit mince et lisse de ciment pur qui avait été appliqué sur la face tendue pout faciliter l'examen. 